\title{
15 Leistungsbewertung der Nachwuchsförderung an Hochschulen: Ein Überblick
}

\subsection{Einleitung}

Im deutschen Hochschulsystem gehört die Nachwuchsförderung zu einer der zentralen Herausforderungen: Einerseits boomt die Wissenschaft gemessen an den Ausgaben, und auch die Anzahl der Nachwuchswissenschaftler/-innen wächst beständig, nämlich um über 75 Prozent seit dem Jahr 2000 (Bundesministerium für Bildung und Forschung 2017). Andererseits fehlt es an langfristigen Perspektiven, insbesondere an Stellen mit unbefristeter Laufzeit. Diese Schieflage hat sich in den letzten Jahren und Jahrzehnten zugespitzt (unter anderem durch die Exzellenzinitiative; Brembs und Welpe, 2019). Sie verlangt zeitnah umfangreiche Veränderungen bei der Nachwuchsförderung im deutschen Wissenschaftssystem, will man nicht weiter an Attraktivität einbüßen. Doch was ist eigentlich Leistung in der Nachwuchsförderung? Und wie lässt sich diese feststellen? Diesen Fragen geht das vorliegende Kapitel nach.

Im Folgenden geben wir einen kurzen Überblick über die aktuelle Situation des wissenschaftlichen Nachwuchses in Deutschland. Anschließend fassen wir Formen der Nachwuchsförderung und deren Messung und Bewertung auf zwei Ebenen zusammen: erstens auf der Makro-Ebene, auf der wir die Nachwuchsförderung mit den Perspektiven in Tätigkeitsbereichen außerhalb der Wissenschaft und deren Veränderungen in den letzten Jahren vergleichen, um so die Attraktivität für eine wissenschaftliche Karriere im Vergleich zu anderen Karrierewegen einordnen zu können, und zweitens auf Meso-, also auf Hochschul-Ebene, auf der wir Leistungskriterien für Hochschulen und Konsequenzen für das Performance-Management gegenüberstellen. Aufbauend auf diesen Betrachtungen formulieren wir Empfehlungen, wie Nachwuchsförderung an deutschen Hochschulen gemessen ${ }^{43}$ und bewertet werden kann. Dies soll als Grundlage dafür dienen, die Nachwuchsförderung an Hochschulen in Deutschland zu verbessern, um damit zu einem leistungsstarken und sozial verantwortlichen Wissenschaftssystem beizutragen.

43 Exakt „messbar“ im Sinne einer naturwissenschaftlichen Vermessung ist die Nachwuchsförderung in Deutschland derzeit nicht, da es bislang kein elaboriertes Messinstrumentarium gibt. Die derzeit vorhandenen Instrumente erlauben aber durchaus Aussagen über ausgewählte Aspekte und auch über die blinden Flecken der bisherigen Versuche - was nachfolgend noch angesprochen werden soll.

René Krempkow, Humboldt-Universität zu Berlin

Jule Specht, Humboldt-Universität zu Berlin

https://doi.org/10.1515/9783110689884-016 


\subsection{Die aktuelle Situation des wissenschaftlichen Nachwuchses}

Über die Begrifflichkeit wissenschaftlicher Nachwuchs ist eine Debatte entbrannt (vgl. BuWiN 2017, S. 65): Wer zählt dazu? Wer noch nicht? Und wer nicht mehr? Im vorliegenden Kapitel zählen zum wissenschaftlichen Nachwuchs all diejenigen, die in der Wissenschaft tätig sind, sich qualifizieren und keine unbefristete Stelle in der Wissenschaft innehaben. Diese sehr inklusiv gedachte Definition des wissenschaftlichen Nachwuchses umfasst daher unter anderem Promovierende, wissenschaftliche Mitarbeiter/-innen ohne Promotion (bis unter 35 Jahren) und mit Promotion (bis unter 45 Jahren), befristet beschäftigte Akademische Rät/-innen, Habilitierende und Juniorprofessor(inn)en sowie Nachwuchsgruppenleitungen (vgl. BuWiN 2017, S. 66).

Der Situation studentischer Hilfskräfte wird im hochschulpolitischen Diskurs beim Thema wissenschaftlicher Nachwuchs vergleichsweise wenig Aufmerksamkeit geschenkt. Dass ihnen in diesem Zusammenhang eine Nebenrolle zugewiesen wird, ist insofern erstaunlich, als dass studentische Hilfskräfte zum einen die nächste Generation an Wissenschaftler(inne)n darstellen und oft während des Studiums für eine wissenschaftliche Karriere angeworben werden (vgl. Bargel und Röhl 2006) und zum anderen tatkräftig dabei unterstützen, den Lehr- und Forschungsbetrieb an Hochschulen aufrechtzuerhalten. Aus diesem Selbstverständnis heraus kämpfen - insbesondere in Berlin - studentische Beschäftigte für Verbesserungen im Tarifvertrag und lehnen eine Aufgabenübertragung in der universitären Verwaltung ab (anstelle in Forschung und Lehre, für die sie eigentlich eingesetzt werden sollen).

Weit mehr Aufmerksamkeit kommt der Situation von Wissenschaftler(inne)n zu, die bereits ihr Studium abgeschlossen haben, aber keine dauerhafte Stelle in der Wissenschaft innehaben. Mittlerweile sind 75 Prozent der wissenschaftlichen Mitarbeiter/-innen an Hochschulen befristet beschäftigt (Vgl. Tab. B10, BuWiN 2017). Unter den jüngeren - den unter 45-Jährigen - sind es sogar 93 Prozent (Vgl. Abb. B36, BuWiN 2017). ${ }^{44}$ Noch gravierender ist die Situation unter den drittmittelfinanzierten Wissenschaftler(inne)n, von denen sogar 98 Prozent befristet beschäftigt sind (Vgl. Tab B10, BuWiN 2017). Angesichts der Situation in der Wirtschaft, wo nur 19 Prozent befristet beschäftigt sind, und ebenfalls deutlich geringeren Anteilen in außeruniversitären Einrichtungen (84 Prozent, mit Promotion 73 Prozent, vgl. BuWiN 2017, S. 127) muss dies auch bei schwankenden (Drittmittel-)Einnahmen aber keineswegs als zwingend gelten. ${ }^{45}$ Obwohl im deutschen Hochschulsystem eine Viel-

44 Vor einer Dekade lag der Anteil deutlich niedriger, so 2005 bei 86 Prozent (BuWiN 2017, S. 127; ähnlich 2013, S. 184).

45 Dies zeigen auch andere europäische Länder mit deutlich geringeren Anteilen an befristet beschäftigten Nachwuchsforschenden, wie die Niederlande mit 40 Prozent oder Norwegen mit 50 Prozent (vgl. Höhle 2015). Ates/Brechelmacher (2013) nennen darüber hinaus (neben den Niederlanden) auch Großbritannien, Irland und Polen als Länder, in denen maximal die Hälfte der Nachwuchsforschenden befristete (Kurzzeit-)Verträge hat. 
zahl an Daueraufgaben bearbeitet werden muss, werden diese selten von Personal übernommen, das dauerhaft angestellt ist. Die durchschnittliche Befristungsdauer lag nach der letzten bundesweiten Erhebung für die Hälfte der Arbeitsverträge bei unter einem Jahr (Jongmanns 2011, BuWiN 2017, S. 30). Zwar gibt es einzelne Hochschulen (z. B. in Berlin), deren durchschnittliche Befristungsdauer inzwischen höher ist. Ein Ende hat die Kettenbefristung jedoch meist erst bei der Berufung auf eine unbefristete Professur - oder nach Weggang aus der Wissenschaft. Berufungen erfolgen in Deutschland im Durchschnitt erst in einem Alter von 40 Jahren, also lange Zeit nach Studienabschluss, der derzeit durchschnittlich mit 24 Jahren erreicht wird (StBA 2018).

Gleichzeitig sind die befristeten Stellen oftmals gering bezahlt. Obwohl viele Doktorand(inn)en quasi Vollzeit und damit regelmäßig mehr Stunden pro Woche als vertraglich vereinbart arbeiten (BuWiN 2017, S. 140 f.), ${ }^{46}$ erhalten diese teilweise ein unterhalb der Armutsgrenze liegendes Gehalt. ${ }^{47}$ Es wird eine Vielzahl von Qualifikationsstellen lediglich mit 50 oder 65 Prozent der regelmäßigen Arbeitszeit vergütet. Besonders deutlich wird das zum Beispiel auch an den Vergütungsmöglichkeiten bei der Deutschen Forschungsgemeinschaft (DFG), die laut DFG-Vordruck 55.02-10/11 unterschiedliche Vergütungsmöglichkeiten je Fach vorsehen. So können in der Geschichtswissenschaft lediglich Stellen mit bis zu 65 Prozent der regulären Arbeitszeit für Promovierende eingeworben werden, beim Maschinenbau aber Stellen mit bis zu 100 Prozent. Unterschiede in der Bezahlung - und damit in der Wertschätzung von Nachwuchswissenschaftler(inne)n machen also deutlich, dass es systematische fachspezifische Unterschiede in der Nachwuchsförderung gibt.

Aus der Situation des wissenschaftlichen Nachwuchses entstehen mindestens zwei zentrale Probleme: Zum einen verliert die wissenschaftliche Karriere durch diese Beschäftigungsbedingungen an Attraktivität, worauf wir nachfolgend noch zurückkommen. Denn durch mangelnde Nachwuchsförderung riskiert man, die besten Köpfe - die man eigentlich für die Wissenschaft gewinnen und halten möchte - an andere Arbeitgeber zu verlieren (vgl. Krempkow 2017a). Wenn aber die Leistungsfähigsten nicht mehr in gewünschtem Ausmaß gewonnen werden können, sinkt das Leistungspotenzial am Wissenschaftsstandort Deutschland. Zum anderen wirkt sich

\footnotetext{
46 Aufgrund der föderal verfassten Bildungslandschaft in Deutschland hängt es vom jeweiligen Landeshochschulgesetz ab, ob die Promotion oder die wissenschaftliche Weiterqualifikation in einem festgelegten Zeitanteil von z. B. einem Drittel in der Arbeitszeit stattfinden kann (so lt. BuWiN 2013, S. 67, in BE, HB, HH, HE, SL, SN und TH), oder nicht.

47 So verfügen in den Sozialwissenschaften und der Geschichtswissenschaft etwas mehr als $15 \mathrm{bzw}$. 20 Prozent der Promovierenden über weniger als 826 Euro im Monat und leben somit unterhalb der Armutsgrenze (vgl. Fräßdorf/Kaulisch/Hornbostel 2012, S. 623). Ähnliches berichtet auch der aktuelle BuWiN (2017, S. 60) insbesondere in Bezug auf Lehrbeauftragte sowie Privatdozent(inn)en, deren Stundenlohn - unter Einberechnung der nicht vergüteten Vor- und Nachbereitung übernommener Lehrveranstaltungen - in der Regel unterhalb des Mindestlohns liegt.
} 
eine mangelnde Nachwuchsförderung auf bestimmte Personengruppen besonders hemmend aus. Und so kommt es, dass unter denjenigen, die die Wissenschaft im Laufe ihrer Karriere verlassen, überproportional viele Frauen sind, viele Menschen mit Eltern ohne Hochschulabschluss sowie Personen mit Migrationshintergrund (vgl. StBA 2017, Krempkow 2017a, 2019).

Zum Thema Leistungsbewertung in der Nachwuchsförderung soll nachfolgend zunächst die Lage in Deutschland insgesamt (Makro-Ebene) bezüglich seiner Entwicklung in den letzten zehn bis 15 Jahren reflektiert werden, bevor danach die Ebene Land-Hochschulen (Meso-Ebene) betrachtet wird.

\subsection{Nachwuchsförderung aus der Makro-Perspektive: Vergleich mit Tätigkeitsbereichen außerhalb der Hochschulen}

Die Leistungsfähigkeit des deutschen Wissenschaftssystems hängt neben den Fördermöglichkeiten in hohem Maße auch von den Möglichkeiten der Rekrutierung junger Wissenschaftler/-innen ab. Denn nicht etwa aus allen, sondern nur aus dem Pool der eine (weitere) Tätigkeit in der Wissenschaft anstrebenden Personen können die Wissenschaftseinrichtungen letztlich ihren Nachwuchs rekrutieren. Hierbei stellt sich anders als in früheren Jahren inzwischen die Frage, inwieweit der wissenschaftliche Nachwuchs noch in die Wissenschaft will. Es geht also „im Kern darum, qualifizierte Wissenschaftler auf allen Stufen der wissenschaftlichen Laufbahn zu attrahieren“ (Peus et al. 2015, S. 4). Deshalb soll anhand von Ergebnissen empirischer Analysen zuerst der Frage nachgegangen werden, inwieweit es derzeit noch gelingen kann, „die Besten für die Wissenschaft zu gewinnen“, wie es u.a. vom Wissenschaftsrat (z. B. 2014) mehrfach gefordert wurde.

Die in den letzten Jahren veröffentlichten Studien zeigen, dass ein großer Teil der Nachwuchsforschenden keineswegs auf das Berufsziel Hochschule beziehungsweise akademische Wissenschaft festgelegt ist (vgl. BuWiN 2017, 2013) und dass sich die Tendenz zum Weggang aus der Wissenschaft verstärkt. So ergab eine vom BMBF geförderte Stifterverbands-Studie, dass erstmals eine Mehrheit aller Nachwuchsforschenden in Deutschland ihre berufliche Zukunft außerhalb der Wissenschaft sieht (vgl. Krempkow et al. 2016, S. 32), während dies zuvor nur für einzelne Gruppen von Nachwuchsforschenden galt.

Wissenschaftseinrichtungen befinden sich damit nicht nur untereinander in einem verstärkten Wettbewerb um die besten Nachwuchsforschenden, sondern unter anderem auch mit Unternehmen der Privatwirtschaft. Dies gilt in besonderem Maße im Hinblick auf Unternehmen mit Forschungs- und Entwicklungsabteilungen, weil diese für forschungsaffine Nachwuchswissenschaftler/-innen attraktiv sind, die wiederum auch das Rekrutierungspotenzial des Hochschullehrer(innen)nachwuchses 
bilden. Ein Spannungsverhältnis zwischen Hochschulen und Unternehmen entsteht allerdings nur, wenn deren Attraktivität als Arbeitgeber nicht zumindest annähernd als gleichwertig wahrgenommen wird. ${ }^{48}$

Insgesamt gaben nach einer jüngsten Erhebung (Krempkow et al. 2016) 39 Prozent der Promovierenden und damit deutlich weniger als 2012 (47 Prozent) an, dass sie beim Einstieg in die akademische Wissenschaft das berufliche Ziel des dauerhaften Verbleibs hatten. Eine andere Frage ist freilich, wie dies auf die Zukunft bezogen aussieht, denn durch die intensivere Befassung mit Forschung, Lehre und wissenschaftlichem Arbeiten ist eine Veränderung sowohl in die eine als auch in die andere Richtung denkbar. Nur weniger als die Hälfte (45 Prozent) gaben an, sie möchten in der akademischen Wissenschaft verbleiben (2012: mit 54 Prozent noch die Mehrheit der Befragten). Hierbei betrifft der Rückgang besonders eine Tätigkeit als Professor/-in (22 Prozent, minus sieben Prozentpunkte), aber in geringerem Ausmaß auch eine Tätigkeit als Wissenschaftler/-in an einer Hochschule oder Forschungseinrichtung jenseits der Professur (23 Prozent, minus zwei Prozentpunkte). Mit 34 Prozent strebten 2015 die meisten Nachwuchswissenschaftler/-innen eine Tätigkeit in der Wirtschaft mit Forschungs- und Entwicklungsbezug an (2012: 29 Prozent). Auch eine Tätigkeit in der Wirtschaft ohne Forschungs- und Entwicklungsbezug wird im Vergleich zu 2012 mit 22 Prozent vermehrt als Ziel genannt (2012: 16 Prozent). ${ }^{49}$

Da zumindest bis vor relativ kurzer Zeit an den Universitäten eine Professur unabhängig von deren Realisierungschance weitgehend als alleiniges Karriereziel für den wissenschaftlichen Nachwuchs galt (bzw. vielerorts noch gilt, vgl. Müller 2017), ${ }^{50}$ sich die Bereitschaft junger Wissenschaftler/-innen hierfür aber in den letzten Jahren deutlich verringerte, ist mit einem eingeschränkten Rekrutierungspotenzial für die Wissenschaftseinrichtungen $\mathrm{zu}$ rechnen. Problematisch ist dies vor allem, wenn die Gründe des Weggangs aus Wissenschaftseinrichtungen darauf hindeuten, dass deren Attraktivität als Arbeitgeber eben nicht zumindest annähernd als gleichwer-

48 An anderer Stelle wird auch der Frage nachgegangen, welche Strategien und Maßnahmen aus Sicht der jeweiligen Einrichtungen relevant sind und Erfolg haben, und es wird thematisiert, wie die Nachwuchsforschenden dies wahrnehmen (Krempkow et al. 2016, S. 48 f.).

49 Natürlich unterscheidet sich dies nach Fächergruppen: So streben 2015 die Befragten der Ingenieurwissenschaften (63 Prozent) und der Mathematik/Informatik/Naturwissenschaften (41 Prozent) am häufigsten eine Tätigkeit in der Wirtschaft mit F\&E-Bezug an. Bei den Geisteswissenschaften ist das Ziel Professur mit 39 Prozent das relativ gesehen noch am häufigsten genannte Ziel; aber selbst hier ist es damit nicht mehr die Mehrheit der Nachwuchsforschenden.

50 Zwar gibt es bereits länger berufliche Orientierungsangebote für den wissenschaftlichen Nachwuchs zu Tätigkeiten außerhalb von Wissenschaftseinrichtungen. Halbwegs flächendeckend angeboten wurde dies jedoch erst seit etwa einem halben Jahrzehnt; 2006 gab es dies noch nicht einmal an einem Drittel der Hochschulen. Zudem ist der Anteil der Hochschulen, die Angebote zur beruflichen Orientierung für Tätigkeiten außerhalb von Wissenschaftseinrichtungen organisieren, zuletzt um sechs Prozentpunkte auf 80 Prozent der Hochschulen gesunken (Krempkow et al. 2016). 
tig mit alternativen Arbeitgebern wahrgenommen wird..${ }^{51}$ Die Ergebnisse zeigen, dass die schlechteren Beschäftigungsperspektiven in der Wissenschaft und die höhere Beschäftigungssicherheit außerhalb der Wissenschaft bei den Nachwuchsforschenden die stärkste Rolle als Grund für einen angestrebten Wechsel spielen. ${ }^{52}$

Für die Attraktivität von Hochschulen für den wissenschaftlichen Nachwuchs (wie für das wissenschaftliche Personal insgesamt) ist es daher auch bedeutsam, inwieweit die Möglichkeit einer unbefristeten Beschäftigung bei entsprechender Leistung vorhanden ist. ${ }^{53}$ Die Stifterverbandsstudie (Krempkow et al. 2016) hat hierzu festgestellt, dass zuletzt an deutlich mehr Hochschulen auch unbefristete Stellen beziehungsweise Tenure-Track-Stellen für Nachwuchswissenschaftler/-innen grundsätzlich möglich sind. ${ }^{54}$ Dies gilt vor allem bei Juniorprofessuren, für die nach der letzten Erhebung an allen Hochschulen auch solche mit Tenure-Track vorhanden sind (2012 waren es nur 64 Prozent der Hochschulen, vgl. Krempkow et al. 2016). Allerdings wurde hierbei nur von 69 Prozent der Hochschulen zumindest grundsätzlich auch „echter Tenure Track“ (mit verbindlicher Entfristung bei Bewährung) angeboten. ${ }^{55}$ Diese Relation wird sich durch das Bund-Länder-Programm für 1.000 TenureTrack-Professuren in den nächsten Jahren zugunsten echter Tenure-Track-Professuren noch verbessern, da deren Schaffung Fördervoraussetzung ist. Für alle anderen Personalkategorien neben der Professur gilt dies weniger (vgl. Krempkow et al. 2016, S. 54): Wenn überhaupt eine Entfristung bei Bewährung vorgesehen ist, dann ist dies bis-

51 In einer DZHW-Studie wurden logistische Regressionen durchgeführt bzgl. des Ziels, eine Tätigkeit außerhalb von F\&L anzustreben. Dabei zeigte sich der stärkste Zusammenhang für die Selbstwirksamkeitserwartung, am zweit- und drittstärksten Karriereorientierung und Arbeitsplatzsicherheit; Elternschaft sowie Akademikerherkunft senken Verbleibswahrscheinlichkeit (Briedis et al. 2014). Das heißt, selbstbewusste, karriereorientierte, langfristig Interessierte, Frauen, Eltern und Akademikerkinder streben eine Beschäftigung signifikant stärker außerhalb der Wissenschaft an (für eine Diskussion dieser Analyse und Einordnung in weitere Ergebnisse vgl. Krempkow et al. 2014).

52 Dagegen spielt „eine höhere Arbeitsbelastung in der Wissenschaft“ als Grund generell nur eine geringe Rolle (für weitere Angaben zu Wechselgründen vgl. Krempkow et al. 2016, S. 35 f.).

53 So hatte auch der Wissenschaftsrat (2014, S. 6) bereits formuliert: „Deutschland braucht ein faires, qualitätsorientiertes und wettbewerbsfähiges Wissenschaftssystem. Dazu gehören adäquate Karriereziele und -wege an Universitäten“. Bereits in früheren Veröffentlichungen hatte er zudem gefordert, dass die Entscheidung über den längerfristigen Verbleib in der Wissenschaft zu einem früheren Zeitpunkt fallen sollte. 2016 hatte auch der sogenannte Imboden-Bericht zur Evaluation der Exzellenzinitiative in Deutschland (vgl. IEKE 2016, S. 28) kritisiert, dass die endgültige Entscheidung über eine akademische Karriere eher in eine spätere Lebensphase verschoben wird, also in die falsche Richtung. 54 Die oftmals geäußerte Befürchtung einer sogenannten „lost generation“ an Nachwuchs-Wissenschaftler(inne)n durch die Einführung von Tenure-Track-Professuren und die damit möglicherweise einhergehende verringerte Zahl an unbefristeten Professuren hat sich bislang nicht bestätigt (Specht/ Kretschmer, 2018).

55 Wichtig ist hierbei natürlich auch, wie viele Personen davon profitieren. Hierzu fanden Schularick et al. (2015) auf Personenebene nur bei 13 Prozent der Juniorprofessuren Tenure-Track-Verträge. Burkhardt et al. (2016, S. 103) berichteten dies von 15 Prozent der Juniorprofessuren. 
lang überwiegend eine unverbindliche (Kann-)Regelung und entspricht damit nicht der Forderung nach Transparenz und Berechenbarkeit. ${ }^{56}$

Letztere zu erfüllen ist aber besonders wichtig, um unerwünschte nicht leistungsbezogene (Selbst-)Selektionseffekte zu vermeiden. Um dies zu veranschaulichen, werden nachfolgend einige Zahlen genannt. Der Gender-Gap in der Wissenschaft wurde bereits häufiger beschrieben (z. B. StBA 2017), deshalb soll dies hier für (Selbst-) Selektionen nach sozialer Herkunft erfolgen: So gibt es deutliche Effekte der sozialen Herkunft zwar bereits beim Zugang zur Hochschule. Aber auch innerhalb des Hochschulsystems hört dies keineswegs auf. So ist der Zugang zur Professur laut den vorliegenden über mehrere Jahrzehnte vergleichbaren Ergebnissen von Möller (2018, S. 266 f.) sogar so sozial selektiv wie noch nie in den letzten 50 Jahren. Hierbei ist die Juniorprofessur (die separat untersucht wurde) zudem besonders sozial selektiv, wie Möller weiter zeigte. Ihre Ergebnisse werden auch durch eine jüngste Studie speziell zu Juniorprofessuren gestützt (vgl. Zimmer 2018). ${ }^{57}$ All dies gilt trotz der an vorgelagerten Schwellen des Bildungssystems von der Grundschule bis zur Promotion existierenden sozialen (Selbst-)Selektionen, die sich allein bereits auf ei-

56 Für eine hohe Attraktivität der Hochschulen als Arbeitsort für hochqualifizierte Nachwuchswissenschaftler/-innen wird allerdings immer wieder die Wichtigkeit hervorgehoben, dass die Personalgewinnung in einem transparenten und (auch) für externe Interessenten offenen Verfahren erfolgt. Erwartungsgemäß sind die Quoten bei Neuberufungen auf eine Professur dem jahrelang maßgeblichen Hausberufungsverbot entsprechend am höchsten. Es ist dennoch bemerkenswert, dass die Quote aktuell nach Angabe der Hochschulen durchschnittlich bereits unter 90 Prozent liegt. An den AuF liegt sie unter 80 Prozent. Damit kommen dort interne Aufstiege mit 22 Prozent etwa doppelt so häufig vor wie an Hochschulen (13 Prozent). Für Postdoc-/Habilitation-/Juniorprofessur/-Nachwuchsgruppenleitungs-Stellen sind es dann durchschnittlich bereits nur noch knapp über die Hälfte (53 Prozent) und an den AuF nur wenig mehr (57 Prozent). Beim Zugang zur Promotion gibt es dagegen einen deutlichen Unterschied zwischen den Einrichtungsarten. An den Hochschulen findet sich der mit Abstand niedrigste Wert externer Personalgewinnung (38 Prozent). Der geringere Anteil an den Hochschulen dürfte v. a. an häufigerer informeller Rekrutierung von Promovierenden liegen (vgl. BuWiN 2008). Zusätzlich kann hier zur Einordnung noch eine Studie der AG Wissenschaftspolitik der Jungen Akademie (vgl. Schularick et al. 2015) zur Berufungspraxis bei Juniorprofessuren herangezogen werden. Sie zeigt, dass bei den meisten deutschen Hochschulen die Quote externer Berufungen zwischen 75 und 90 Prozent liegt. Allerdings gebe es auch große Institutionen wie die FU Berlin mit 67 Prozent oder die LMU München mit nur 56 Prozent externen Berufungen, sodass die Konsequenzen einen relativ hohen Anteil der Juniorprofessuren beträfen, heißt es dort weiter (vgl. Schularick et al. 2015, S. 7).

57 Dabei ist in Anlehnung an Bourdieu (1992) für den Berufungserfolg v. a. soziales Kapital ein starker Einflussfaktor und nicht etwa wissenschaftliches Kapital: Wichtige Einflussfaktoren auf den Berufungserfolg sind demnach im Einzelnen v. a. Kontakte in die Professorenschaft und Aufenthalt(e) an Universitäten der Ivy League (USA) oder des Golden Triangle (UK). Als eigenes wissenschaftliches Kapital bzw. als Leistungskriterien wahrgenommene Einflussfaktoren wie Zeitschriftenartikel mit Peer Review, Drittmittelprojekte oder Konferenzbeiträge hatten dagegen keine statistisch nachweisbaren Effekte auf den Berufungserfolg (ausführlich vgl. Zimmer 2018, S. 262, zur Einordnung in frühere Studien vgl. Krempkow 2019). 
ne Chancenrelation von 10:1 zugunsten von Akademikerkindern aufsummieren (vgl. Stifterverband und McKinsey 2017). Daraus kann abgeleitet werden: Ein leistungsfähiges nationales System der Nachwuchsförderung hat nicht nur „den Auftrag, die besten Talente unabhängig vom Geschlecht für die Wissenschaft zu gewinnen“ (vgl. Peus et al. 2015, S. VII), sondern auch unabhängig von anderen Aspekten wie der (sozialen) Herkunft.

Selbst auf der Ebene der Hochschulleitungen spricht die Homogenität der Präsident(inn)en und Rektor(inn)en noch für starke Einflüsse von (Selbst-)Selektionen: So wurden einer Studie von Roessler (2019) zufolge 95 Prozent dieses Spitzenpersonals in Deutschland geboren, aber keine einzige Person davon in den neuen Bundesländern, obwohl diese etwa 20 Prozent der Bürger/-innen ausmachen.

Dabei sind die Leistungen des deutschen Hochschulsystems in der Nachwuchsförderung gemessen an zentralen Zahlen aus dem BuWiN (2017) durchaus beachtlich: So werden dort unter „Bestandsdaten zum wissenschaftlichen Nachwuchs“ deutliche Steigerungen der Zahl der Promovierenden und Promovierten sowie der Zahl der Nachwuchsgruppenleitungen berichtet, die Habilitationen sind in den letzten Jahren zurückgegangen. ${ }^{58}$

Darüber hinaus wird im BuWiN-Abschnitt „Leistungen des wissenschaftlichen Nachwuchses und Wirkungen der wissenschaftlichen Qualifizierung“ zunächst die internationale Mobilität des wissenschaftlichen Nachwuchses betrachtet. Zwar wird auch der Beitrag des wissenschaftlichen Nachwuchses zu Forschung, Lehre und Transfer annähernd quantifiziert beziehungsweise Möglichkeiten für entsprechende Analysen benannt. Allerdings zeigen sich - anders als zum vergleichsweise gut darstellbaren Beitrag für die Lehre ${ }^{59}$ - in den Bereichen Forschung und Transfer erhebliche Daten- und Forschungslücken (vgl. BuWiN 2017, S. 23 f.).

Für die Weiterentwicklungen des Berichtswesens zu den Leistungen hat eine Expertengruppe unter Leitung des Statistischen Bundesamtes ein Indikatormodell für die Berichterstattung zum wissenschaftlichen Nachwuchs (StBA 2014) entwickelt, das zugleich Anregungen für die Weiterentwicklung von Datenerhebungen mittels Befragungen von Forschungseinrichtungen sowie der amtlichen Statistik gibt. Das Indikatormodell benennt Indikatoren für Promovierende (z. B. Anzahl, Promotionsabbrüche) und für Promovierte (z. B. Anzahl, Erwerbssituation, Beschäftigungsbedingun-

58 Darüber hinaus wird dies u. a. auch nach soziodemografischen Kategorien wie Fächergruppenzugehörigkeit, Frauenanteil und Durchschnittsalter ausgewiesen sowie anhand von Promotionsquoten als Anteil der Promotionen an den Hochschulabschlüssen. Einige relative Indikatoren wie die Relation Promotionen je Professur werden im BuWiN (2017) anders als im BuWiN (2008) allerdings nicht mehr ausgewiesen.

59 So sind beispielsweise 67 Prozent der Promovierenden an Universitäten in die Lehre eingebunden. An Fachhochschulen sind es 65 Prozent und bei außeruniversitären Forschungseinrichtungen 17 Prozent. Sie lehren im Schnitt 4,2 Semesterwochenstunden (vgl. BuWiN 2017, S. 35). 
gen) sowie karrierephasenübergreifende Indikatoren (z. B. Anzahl Forscher/-innen, Hochschulpersonal), mit denen sich der Anspruch verbindet, anschlussfähig an die nationale und internationale Bildungs- und Forschungsberichterstattung zu sein und sich möglichst auf regelmäßig erhobene amtliche und nicht amtliche Datenquellen zu stützen. Neben der Darstellung wichtiger existierender Daten wurden im Bericht zudem Indikatoren für Bereiche entwickelt, für die bisher noch keine Kennziffern zur Verfügung stehen. Vorhandene Datenlücken wichtiger Kennziffern wurden beschrieben, Strategien zur Datengewinnung skizziert.

Darüber hinaus wurden mit den Empfehlungen des Wissenschaftsrats (2016) zu einem Kerndatensatz Forschungsbereiche vorgeschlagen, für die Hochschulen und Forschungseinrichtungen Daten vorhalten sollten. Für diese Bereiche wurden Kerndaten identifiziert, die als essenziell erachtet werden und für die Vorschläge zur Standardisierung konsentiert werden konnten. Ab dem Jahr 2020 soll es zudem möglich sein, mittels Promovierendenerfassung im Regelbetrieb Erfolgs- bzw. Abbruchquoten für Promotionen zu berichten.

Eine relevante Orientierungsgröße für ein Monitoring der Entwicklungen im Hochschulsystem ebenso wie für Nachwuchsforschende mit Interesse an einer Wissenschaftskarriere wäre auch die Wahrscheinlichkeit einer Berufung auf unbefristete Professuren. Allerdings schwanken allein die im Jahr 2017 in öffentlichen Diskussionen genannten Zahlen zu Berufungschancen von 1:300, wie sie der Präsident der Hochschulrektorenkonferenz Peter-André Alt nannte, bis zu etwa 1:1 vom ehemaligen Präsidenten der Leibniz-Gemeinschaft und BuWiN-Beiratsvorsitzenden, Karl Ulrich Mayer (vgl. Mayer 2017). Im BuWIN selbst (2017, S. 194) finden sich einige fächerunspezifische Zahlen zu Berufungschancen aus einer GWK-Analyse. Diese zeigen für 2014 das Verhältnis von insgesamt 45.378 Bewerbungen zu insgesamt 2.007 erfolgreichen Berufungen, also eine Relation von 1:24 (oder 4 Prozent). ${ }^{60}$ Ein Problem bei diesen Zahlen zu den Berufungschancen ist aber, dass sie aus der Vergangenheit direkt auf die Zukunft schließen, ohne die voraussichtlich frei werdenden Professuren zu berücksichtigen. Außerdem wäre für eine Orientierungsfunktion eine Analyse zumindest nach Fächergruppen notwendig, welche mit den verfügbaren Daten grundsätzlich möglich wäre und insbesondere für die Ingenieurwissenschaften um ein Mehrfaches günstigere Chancen aufzeigt als für andere Fächergruppen (vgl. Berechnungsbeispiele in Krempkow 2017b).

60 Allerdings wurden dort Neuberufungen und Weg-Berufungen (von Professurinhabern) sowie Juniorprofessuren (bis dahin fast immer ohne echten Tenure Track) zusammengefasst. Neuberufungen auf Dauerstellen waren laut BuWiN (2017, S. 191) im Jahr 2014 aber nur 872, d. h.: Durchschnittlich nur jede 52. Bewerbung (oder rund zwei Prozent aller Bewerbungen) war zuletzt erfolgreich im Sinne eines dauerhaften Verbleibs in der Wissenschaft. 


\subsection{Nachwuchsförderung aus der Meso-Perspektive: Leistungskriterien innerhalb der Hochschulen}

\subsubsection{Die Nachwuchsförderung in der Landes-Hochschul-Berichterstattung}

Bereits vor über zehn Jahren wurden in Landeshochschulberichten relativ umfassende Indikatorsysteme für die Nachwuchsförderung an einzelnen Hochschulen entwickelt, in denen sowohl absolute als auch relative Indikatorwerte nach Fächergruppen gegenüberstellt wurden, so z. B. im bereits seit 2006 regelmäßig erstellten Hochschulbericht Sachsen (Lenz et al. 2006). Hierbei werden (neben Forschungsindikatoren wie Drittmitteln) als Indikatoren für die Nachwuchsförderung einerseits die Anzahl der abgeschlossenen Promotionen und Habilitationen sowie Neuberufungen ${ }^{61}$ nach Fachrichtungen und Fächergruppen an den einzelnen Hochschulen berichtet, andererseits aber auch die Relation Promotionen und Habilitationen je Professur, um eine Vergleichsmöglichkeit auch mit bundesweiten Daten zu schaffen. ${ }^{62}$ Seit etlichen Jahren gibt es in anderen Bundesländern wie z. B. Berlin ähnliche Indikatoren, die aber aufgrund ihrer Kopplung mit der Hochschulfinanzierung nicht als Berichtssystem, sondern als Anreizsystem fungieren.

Bezüglich dieser Indikatorwerte gibt es jedoch Verbindungen zur PerformanceMeasurement-Diskussion: So weist Klumpp (in Welpe et al. 2015, S. 432 f.) darauf hin, dass viele Hochschulen nur wenige Indikatoren wie z. B. Anzahl der Publikationen, Patente, Drittmittel und (Promotions-)Abschlüsse für ein eindimensionales Performance-Measurement verwenden, wo für eine adäquate Erfassung ihrer Leistungen „multiple input and multiple output measurements“ notwendig wären (Welpe et al. 2015, S. 433). Insbesondere fehlt es nach Ringelhan (in Welpe et al. 2015, S. 87 f.) bislang in Performance-Management-Modellen noch an einer besseren Erfassung von Qualitätsunterschieden. Aus konzeptioneller Sicht wären zudem noch Indikatoren für die Attraktivität von Hochschulen aus Sicht von Nachwuchswissenschaftler(inne)n wünschenswert (vgl. z. B. Promovierenden-Initiative 2002).

\footnotetext{
61 Juniorprofessuren und Nachwuchsgruppenleitungen spielten zum damaligen Zeitpunkt quantitativ keine Rolle.

62 In geschlechterdifferenzierenden Auswertungen zeigte sich, dass die Ergebnisse insbesondere in den höheren Qualifikations- bzw. Hierarchiestufen auch mit einer überdurchschnittlich starken Selektivität nach Geschlecht zusammenhängen (vgl. Lenz et al. 2006, S. 479 f.), welche wiederum mit für Frauen überdurchschnittlich häufigen prekären Beschäftigungsverhältnissen einhergehen. Darüber hinaus wurden u. a. die rechtlichen Rahmenbedingungen der Nachwuchsförderung (vgl. auch BuWiN 2008, S. 100) sowie die berufliche Autonomie und Zukunftsperspektiven zur Einordnung der Ergebnisse herangezogen.
} 


\subsubsection{Die Nachwuchsförderung in der leistungsorientierten Mittelverteilung}

Wissenschaftlicher Nachwuchs ist in vielen Modellen der leistungsorientierten Mittelverteilung auf der Ebene Land-Hochschule ein wesentliches Kriterium, von dem die Zuweisung der Mittel an die einzelnen Hochschulen abhängt. Bereits 2008 hatten nur die Modelle in Hamburg und Bremen keinen „Nachwuchsindikator“. Erfasst wird die Nachwuchsförderung der einzelnen Hochschulen in der Regel anhand der abgeschlossenen Promotionen oder anhand einer Promotionsquote wie z. B. Promotionen pro Professur (vgl. König 2011, Krempkow 2012). ${ }^{63}$ Die Gewichtung dieser Indikatoren zur Nachwuchsförderung war gemessen an deren Anteil im Gesamtbudget insgesamt nicht sehr hoch (0,16 bis 7,5 Prozent, vgl. BuWiN 2008, S. 120). Neben diesen allein auf die Nachwuchsförderung bezogenen Indikatoren können aber auch Indikatoren zur Chancengerechtigkeit und zur Herkunft der Wissenschaftler/-innen aufgrund ihres Querbezugs zur Nachwuchsförderung von Bedeutung sein. Beispielsweise flossen im Land Berlin bereits vor Jahren neben den Frauenanteilen der Graduierten auch die der Promovierten und Neuberufenen in die Gleichstellungsquote ein. Ähnlich gibt es dies auch für ausländische Wissenschaftler/-innen als Indikator für das Ausmaß der Internationalisierung (vgl. Breitbach 2009, Krempkow et al. 2013). In den Folgejahren spielte dies eine ähnliche Rolle (vgl. auch Butler 2010). So heißt es im zweiten BuWiN (2013), dass sich in 14 von 15 Modellen zur leistungsorientierten Mittelverteilung zwischen Ländern und Universitäten die Zahl der abgeschlossenen Promotionen positiv auf das Budget der Universitäten auswirkt.

Auch wenn im jüngsten BuWiN (2017) zur Nachwuchsförderung als Gegenstand von Zielvereinbarungen und leistungsorientierter Mittelverteilung (LoM) keine Informationen mehr geliefert wurden, können wir davon ausgehen, dass dies in der Hochschulsteuerung auch weiter eine Rolle spielt. Allerdings war zu einzelnen Bundesländern wie z. B. Baden-Württemberg überregionalen Presseberichten zu entnehmen, dass sie die Anzahl der Promotionen nicht mehr in der LoM berücksichtigen, um Fehlanreize zur Erhöhung der „Stückzahl“ zu vermeiden (vgl. zu solchen Fehlanreizen ausführlicher auch Krempkow et al. 2007, 2013).

Für die LoM innerhalb von Hochschulen und innerhalb von Fakultäten sind leider in Deutschland bislang nur wenige systematische Erhebungen verfügbar. Die vorliegenden Informationen zeigen, dass Indikatoren zur wissenschaftlichen Nachwuchsförderung auf dieser Ebene auch relativ häufig genutzt werden und mit ähnlicher Gewichtung in Berechnungsformeln einfließen wie in Landesmodellen (z. B. Bogumil et al. 2011, Jochheim und Bogumil 2015). So war nach einer Erhebung bei 55 Leitungen von Forschungsgruppen an Universitäten schon in den Jahren ab 2006 die LoM bereits bei vier Fünfteln der Befragten eingeführt worden (Jansen 2007). Bei einem großen

63 Ein Überblick zu zwölf Bundesländern findet sich im ersten BuWiN (2008: 120), detaillierte Beschreibungen von fünf Landesmodellen in Breitbach (2009). Neuere Publikationen des Kanzlerarbeitskreises Hochschulfinanzierung gingen bislang nicht ausführlicher auf die LoM ein. 
Teil davon war auch die Anzahl der abgeschlossenen Promotionen ein Indikator. ${ }^{64}$ In der Hochschulmedizin wurde die LoM bereits ab 2005 in allen Medizinischen Fakultäten in Deutschland eingeführt. An einem Viertel davon wurden neben Drittmitteln und Publikationen weitere Kriterien genutzt, hier v. a. Habilitation als Indikator der Nachwuchsförderung. Deren Gewichtung innerhalb der Forschungs-LoM variierte allerdings erheblich: Sie lag zwischen sieben und 60 Prozent, was gemessen an den im BuWiN (2008) berichteten Gewichtungen als relativ hoch gelten kann. Hier gibt es aus Sicht der Forschenden durch die LoM zwar Transparenz- und Effizienzsteigerungen, aber zugleich auch vermehrt Mainstream-Forschung und „Einzelkämpfertum“ (vgl. Krempkow et al. 2013).

Für die existierenden Performance-Management-Modelle werden insgesamt in der Literatur verschiedene nicht intendierte Effekte konstatiert (vgl. Überblick von Ringelhan et al. in Welpe et al. 2015, S. 96 f.). So weisen Ringelhan et al. beispielsweise auf Verdrängungseffekte der intrinsischen Motivation durch Leistungsanreize hin sowie Bezug nehmend auf den Wissenschaftsrat (2011) auf die Gefahr der Vernachlässigung der Lehre. Eine Herausforderung für die Zukunft liegt daher in einer besseren Vermeidung von nicht intendierten Effekten. Ansatzpunkte hierfür wären ideelle Leistungsanreize, die Reputation fördern, wie z. B. geeignete Preise und Auszeichnungen (vgl. Krempkow in Welpe et al. 2015, S. 207 f.), oder andere nicht monetäre Anreize, die intrinsische Motivation durch größere Freiräume sowie durch die Autonomie von (Nachwuchs-)Forschenden fördern. So könnte über die Verbesserung der Rahmenbedingungen und der beruflichen Zufriedenheit letztlich deren Leistungen verbessert werden (vgl. zu solchen Ansätzen auch Krempkow 2007).

\subsection{Ausblick und Empfehlungen}

Die vorgestellten Ergebnisse haben gezeigt, dass für Nachwuchsforschende die schlechteren Beschäftigungsperspektiven in der Wissenschaft und die höhere Beschäftigungssicherheit außerhalb der Wissenschaft die stärkste Rolle für die Entscheidung zum Weggang aus der akademischen Wissenschaft spielen. Hierauf kann und sollte mit verstärkten Angeboten zur Schaffung von Transparenz und Berechenbarkeit sowie einer früheren Entscheidung über den längerfristigen Verbleib in der Wissenschaft reagiert werden, was mit Tenure-Track-Angeboten zunehmend auch adressiert wird. Darüber hinaus sollte aber auch auf eine Minderung sozialer (Selbst-) Selektion fokussiert werden, um aus dem vollen Leistungspotenzial für den Wissenschaftsstandort schöpfen zu können.

64 Zwar wird nach Jochheim/Bogumil (2015) mehrheitlich die psychologische Wirkung, die von einer transparenten Formel ausgeht, hervorgehoben und als stärker eingeschätzt als deren materieller Anreiz. Insgesamt wird jedoch zu bedenken gegeben, dass man durch eine Formel nur quantitative Faktoren berücksichtigen könnte, was zwangsläufig dazu führen würde, dass die Qualität der Leistungen darunter leide bzw. falsche Anreize gesetzt würden. 
Wie an anderer Stelle bereits dargestellt, sehen die Wissenschaftseinrichtungen wie auch die Wissenschaftspolitik - bereits die Notwendigkeit, dem wissenschaftlichen Nachwuchs mit abgeschlossener Promotion in verschiedenen Personalkategorien mehr Möglichkeiten einer unbefristeten Beschäftigung anzubieten, und sie planen eine moderate, aber spürbare Erhöhung der Entfristungen (vgl. Krempkow et al., 2016). Wichtig ist hierbei jedoch die Berechenbarkeit der Chancen auf eine höhere Beschäftigungssicherheit, damit dies bei nachgewiesener Leistung nicht länger z. T. vom zufälligen Freiwerden von Stellen abhängt. Die Berechenbarkeit kann durch strikte Transparenz und Verbindlichkeit sowie durch vorab festgelegte Kriterien für die Stellenbesetzung (vgl. Peus et al. 2015, S. 8 f.) gefördert werden, so wie es auch für das Bund-Länder-Programm für die Tenure-Track-Professuren gefordert wird. Diese Grundsätze sollten für Entfristungen anderer Personalkategorien neben der Professur ebenfalls gelten, damit auch sie nicht von zufällig frei werdenden Haushaltsstellen abhängen. Konkret könnte das z. B. für Senior-Scientist-Positionen ähnlich ablaufen wie bei Zwischenevaluationen für das Emmy-Noether-Programm der DFG, was in einzelnen Wissenschaftseinrichtungen bereits erfolgte. Für Wissenschaftsmanagement-Stellen könnte dies z. B. über Zielvereinbarungen zur Entfristung erfolgen, wie an einzelnen Hochschulen bereits gehandhabt. Aber auch die Chancen auf eine Berufung könnten sowohl für Wissenschaftseinrichtungen als auch für Nachwuchsforschende noch berechenbarer gestaltet werden, z. B. indem standardmäßig vorab ein Anforderungsprofil inklusive konkreter Aufgaben einer Stelle erstellt wird.

Darüber hinaus könnte die Berechenbarkeit der Verbleibchancen auch über einzelne Wissenschaftseinrichtungen hinaus noch verbessert werden, indem zum Beispiel eine Aufbereitung von Daten für ein Monitoring der Berufungschancen erfolgt. Derzeit werden Berufungschancen für Promovierte sehr unterschiedlich angegeben und eine fächerspezifische Berechnung auf Basis der voraussichtlich freiwerdenden Professuren liegt nicht vor. Auf längere Sicht könnten daher nicht nur Transparenz und Verbindlichkeit sowie vorab festgelegte Kriterien, sondern auch eine bessere Datenbasis und -aufbereitung die Berechenbarkeit der Berufsperspektiven in der Wissenschaft verbessern.

Insgesamt könnte dies helfen, Wissenschaftseinrichtungen und Nachwuchsforschenden validere Entscheidungsgrundlagen für oder gegen den Verbleib im Wissenschaftssystem zu ermöglichen und letztlich aus einem größeren und passenderen Rekrutierungspotenzial die (bzw. mehr von den) Besten zu gewinnen.

\subsection{Literaturverzeichnis}

Ates, G. und Brechelmacher, A. (2013). Academic Career Paths. In U. Teichler und E. A. Höhle (Hrsg.), The Work Situation of the Academic Profession in Europe: Findings of a Survey in Twelve Countries. The Changing Academy - The Changing Academic Profession in International Comparative Perspective 8, S. 13-35, Dordrecht u. a. 
Bargel, T. und Röhl, T. (2006). Wissenschaftlicher Nachwuchs unter den Studierenden. Empirische Expertise auf der Grundlage des Studierendensurveys. Bonn, Berlin: Bundesministerium für Bildung und Forschung.

Bogumil, J., Heinze, R. G. und Gerber, S. (2011). Neue Steuerung von Universitäten - Erste Ergebnisse der deutschlandweiten Befragung. Workshop „Neue Steuerung von Universitäten“, RuhrUniversität Bochum.

Breitbach, M. (2009). Empfehlungen zur Gestaltung von Steuerungssystemen. Kanzlerarbeitskreis „Leistungsorientierte Mittelvergabe und Zielvereinbarungen“.

Bundesministerium für Bildung und Forschung (2017). Gute Perspektiven für den wissenschaftlichen Nachwuchs: Wie die Bundesregierung Karrierewege junger Wissenschaftlerinnen und Wissenschaftler unterstützt. Berlin.

Butler, L. (2010). Impacts of Performance-Based Research Funding Systems: A review of the concerns and the evidence. In Performance-Based Funding for public research in tertiary education institutions, workshop proceedings, S. 127-166. Brussels: OECD publishing.

BuWiN (2008). Bundesbericht zur Förderung des Wissenschaftlichen Nachwuchses (BuWiN). Bonn, Berlin: BMBF.

BuWiN (2013). Konsortium Bundesbericht Wissenschaftlicher Nachwuchs 2013. Statistische Daten und Forschungsbefunde zu Promovierenden und Promovierten in Deutschland. Bielefeld.

BuWiN (2017). Konsortium Bundesbericht Wissenschaftlicher Nachwuchs. Statistische Daten und Forschungsbefunde zu Promovierenden und Promovierten in Deutschland. Bielefeld.

Fräßdorf, A., Kaulisch, M. und Hornbostel, S. (2012). Armut und Ausbeutung? Die Finanzierungs- und Beschäftigungssituation von Promovierenden. Forschung \& Lehre, 8/2012:622-624.

Höhle, E. (2015). From Apprentice to Agenda-Setter: Comparative Analysis of the Influence of Contract Conditions on Roles in the Scientific Community. Studies in Higher Education, 40(8):1-15.

IEKE (2016). Internationale Expertenkommission zur Evaluation der Exzellenzinitiative. Endbericht. Januar 2016.

Jansen, D. H. (2007). New Forms of Governance in Reserach Organizations. Disciplinary Approaches, Interfaces and Integration. Dordrecht.

Jochheim, L. und Bogumil, J. (2015). Wirkungen neuer Steuerungsinstrumente auf die Aktivitätsstruktur von Universitäten - Leistungsorientierte Mittelvergabe, Zielvereinbarungen und W-Besoldung im Fallstudienvergleich. Hochschulmanagement (HM), 3+4/2015:106-112.

Jongmanns, G. (2011). Evaluation des Wissenschaftszeitvertragsgesetzes (WissZeitVG). Gesetzesevaluation im Auftrag des Bundesministeriums für Bildung und Forschung. HIS: Forum Hochschule, 4/2011.

König, K. (2011). Hochschulsteuerung. In P. Pasternack (Hrsg.), Hochschulen nach der Föderalismusreform, S. 106-154. Leipzig: Akademische Verlagsanstalt.

Krempkow, R. (2019). Wieviel zählt Leistung bei Berufungen, und wieviel Herkunft? Qualität in der Wissenschaft (QiW), 1/2019:28-31.

Krempkow, R. (2017a). Können wir die Besten für die Wissenschaft gewinnen? Zur Rekrutierung von Nachwuchsforschenden in Wissenschaft und Wirtschaft. Personal- und Organisationsentwicklung (P-OE), 2+3/2017:59-64.

Krempkow, R. (2017b). Was kann die aktuelle Forschung über Berufungschancen sagen? Anmerkungen zur Schätzung von Karl-Ulrich Mayer. Forschung, 2/2017:66-70.

Krempkow, R., Sembritzki, T., Schürmann, R. und Winde, M. (2016). Personalentwicklung für den wissenschaftlichen Nachwuchs 2016. Bedarf, Angebote und Perspektiven - eine empirische Bestandsaufnahme im Zeitvergleich. Berlin: Stifterverband.

Krempkow, R. und Landrock, U. (2013). Wie effektiv ist die Leistungsorientierte Mittelvergabe? Zwischenbilanz ein Jahrzehnt nach ihrer Einführung in der Hochschulmedizin. In E. Grande, D. Jansen, O. Jarren, A. Rip, U. Schimank und P. Weingart (Hrsg.), Neue Governance der Wissenschaft: Reorganisation, Externe Anforderungen, Medialisierung, S. 95-111, Bielefeld. 
Krempkow, R., Landrock, U., Neufeld, J. und Schulz, P. (2013). Intendierte und nicht-intendierte Effekte dezentraler Anreizsysteme am Beispiel der fakultätsinternen leistungsorientierten Mittelvergabe in der Medizin. Abschlussbericht des Projektes GOMED - Governance Hochschulmedizin. Berlin.

Krempkow, R. (2012). Kann mit leistungsorientierter Mittelvergabe die Chancengerechtigkeit für Nachwuchswissenschaftler/innen mit Migrationshintergrund gefördert werden? Die Hochschule, 1/2012:143-153.

Krempkow, R. (2007). Leistungsbewertung, Leistungsanreize und die Qualität der Hochschullehre. Konzepte, Kriterien und ihre Akzeptanz. Bielefeld.

Lenz, K., Krempkow, R. und Popp, J. (2006). Sächsischer Hochschulbericht 2006. Dauerbeobachtung der Studienbedingungen und Studienqualität im Freistaat Sachsen. Sächs. Kompetenzzentrum für Bildungs- und Hochschulplanung der TU Dresden.

Mayer, K. U. (2017). Zur Lage junger Wissenschaftlerinnen und Wissenschaftler im deutschen Wissenschaftssystem. Forschung, 1/2017:31-35.

Müller, M. (2017). Karriere nach der Wissenschaft. Alternative Berufswege für Promovierte. Frankfurt a. M.

Peus, C., Braun, S., Hentschel, T. und Frey, D. H. (2015). Personalauswahl in der Wissenschaft. Evidenzbasierte Methoden und Impulse für die Praxis. Berlin/Heidelberg.

Promovierenden-Initiative (2002). Statistische Kennzahlen für den Prozess des Promovierens. Arbeitspapier der Promovierenden-Initiative (PI).

Rogge, J.-C. (2017). Karrierewege und Orientierungen in Wirtschaft und Wissenschaft im Vergleich Ausdifferenzierung vs. Zuspitzung? WSI-Mitteilungen, 05/2017:364-371.

Roessler, I. (2019). Universitätsleitung in Deutschland. Gütersloh: CHE gemeinnütziges Centrum für Hochschulentwicklung.

Schularick, M., Specht, J. und Baumbach, S. (2015). Berufungspraxis bei Juniorprofessuren in Deutschland, Studie der AG Wissenschaftspolitik der Jungen Akademie. Berlin.

Specht, J. und Kretschmer, R. (2018). Eine verlorene Generation scheint es nicht zu geben. FAZ. https://www.faz.net/aktuell/karriere-hochschule/akademischer-mittelbau-eine-verlorenegeneration-scheint-es-nicht-zu-geben-15911044.html (Zugriff am 06.01.2020).

StBA (2014). Statistisches Bundesamt: Indikatorenmodell für die Berichterstattung zum wissenschaftlichen Nachwuchs. Endbericht. Wiesbaden.

StBA (2018). Statistisches Bundesamt: Durchschnittsalter von Hochschulabsolventen in Deutschland von 2003 bis 2016 (in Jahren). Statista. https://de.statista.com/statistik/daten/studie/ 189237/umfrage/durchschnittsalter-von-hochschulabsolventen-in-deutschland/ (Zugriff am 2.3.2018).

StBA (2017). Statistisches Bundesamt: Frauenanteile Akademische Laufbahn. Verfügbar unter: www.destatis.de/DE/ZahlenFakten/GesellschaftStaat/BildungForschungKultur/Hochschulen/ Tabellen/FrauenanteileAkademischeLaufbahn.html (Zugriff am 21.02.2018).

Stifterverband und McKinsey (2017). Hochschul-Bildungs-Report 2020: Höhere Chancen durch höhere Bildung? Jahresbericht 2017/18 - Halbzeitbilanz 2010 bis 2015. Berlin: Stifterverband für die Deutsche Wissenschaft e. V.

Welpe, I., Wollersheim, J., Ringelhan, S. und (Hrsg.), O. M. (2015). Incentives and Performance: Governance of Knowledge-Intensive Organizations. München.

Wissenschaftsrat (2014). Empfehlungen zu Karrierezielen und -wegen an Universitäten.

Wissenschaftsrat (2011). Empfehlungen zur Bewertung und Steuerung von Forschungsleistungen. Drs. 1656-11. Halle: Wissenschaftsrat. 